\title{
Fuel-Efficient Trajectories Traffic Synchronization
}

\author{
Adrian Pawełek* and Piotr Lichota \\ Warsaw University of Technology, 00-665 Warsaw, Poland \\ and \\ Ramon Dalmau $\$$ and Xavier Prats $\underline{\S}$ \\ Technical University of Catalonia, 08860 Castelldefels, Spain
}

DOI: $10.2514 / 1 . C 034730$

\begin{abstract}
Continuous descent operations (CDOs) with required times of arrival (RTA) have been identified as a potential solution for reducing the environmental footprint of aviation in the terminal maneuvering area without compromising capacity. This paper assesses the feasibility of replacing current air traffic control sequencing and merging techniques, mainly based on path stretching and air holding, by a control based on RTA over metering fixes on known and fixed arrival routes. Because the remaining distance to the runway threshold is always known by the aircraft crew, this would allow engine-idle CDOs that do not require speed-brake usage and where only elevator control is used to meet the RTA. The assessment has been performed for Barcelona-El Prat Airport (Spain) using historical traffic demand data. The earliest and latest trajectories at a metering fix for each inbound aircraft were computed assuming engine-idle CDOs. Given the attainable RTA window for each aircraft, the aircraft sequencing problem was solved. The results show that assigning RTA allows optimizing the landing sequence when air traffic is low. For scenarios with high-traffic loads and late RTA assignments, path stretching still was found to be necessary. The minimum distance from the runway where inbound aircraft should receive the RTA to fully remove any radar vectoring was also analyzed. It was demonstrated that the assignment of RTA well before starting the descent would favor to enable full CDOs.
\end{abstract}

\section{Nomenclature}

$\begin{array}{ll}\boldsymbol{A}, \boldsymbol{P} & =\text { sets of aircraft and of aircraft pairs, respectively } \\ B & =\text { binary variable } \\ D & =\text { aerodynamic drag, } \mathrm{N} \\ g & =\text { gravity acceleration, } \mathrm{m} / \mathrm{s}^{2} \\ h & =\text { altitude, ft } \\ J & =\text { cost function } \\ L & =\text { positive scalar variable } \\ M & =\text { Mach number } \\ m & =\text { mass of the aircraft, kg } \\ p, q & =\text { positive integer variables } \\ p, \boldsymbol{u}, \boldsymbol{x} & =\text { parameters, control, and state vectors } \\ S & =\text { Airbus A320 specific speed, kt } \\ s & =\text { along path distance, } \mathrm{n} \text { mile } \\ T & =\text { aircraft thrust, } \mathrm{N} \\ t & =\text { time, } \mathrm{s} \\ v & =\text { true airspeed, kt } \\ v_{\mathrm{CAS}} & =\text { calibrated airspeed, kt } \\ \gamma & =\text { aerodynamic flight-path angle, deg } \\ \Delta t_{\text {sep }} & =\text { time separation, } \mathrm{s} \\ \Delta t_{+}, \Delta t_{-} & =\text {positive scalar variables }\end{array}$

\section{Introduction}

$\mathbf{A}^{\top}$ T PRESENT, air traffic controllers (ATCs) use tactical instructions, such as speed adjustments, altitude assignments, and path stretching (also known as radar vectoring), and eventually air

Presented as Paper 2017-3770 at the 17th AIAA Aviation Technology, Integration, and Operations Conference, Denver, CO, 5-9 June 2017; received 29 August 2017; revision received 24 July 2018; accepted for publication 28 July 2018; published online 12 October 2018. Copyright (C) 2018 by the American Institute of Aeronautics and Astronautics, Inc. All rights reserved. All requests for copying and permission to reprint should be submitted to CCC at www.copyright.com; employ the eISSN 1533-3868 to initiate your request. See also AIAA Rights and Permissions www.aiaa.org/randp.

*Ph.D. Student, Institute of Theoretical and Applied Mechanics; apawelek@meil.pw.edu.pl.

${ }^{\dagger}$ Associate Professor, Institute of Theoretical and Applied Mechanics; plichota@meil.pw.edu.pl.

‡Ph.D. Student; ramon.dalmau@upc.edu.

${ }^{\S}$ Associate Professor; xavier.prats@upc.edu. holding, to maintain safe separation between arriving and departing aircraft in the terminal maneuvering area (TMA). These instructions usually degrade the performance of climb and descent operations, leading to higher-than-necessary fuel consumption, gaseous emissions, and noise nuisance.

The reduction of the environmental impact of aviation is one of the major drivers of current research efforts in air transportation. It was already demonstrated that significant environmental impact mitigation in the TMA can be achieved by using continuous descent operations (CDOs) [1-3]. This approach consists of descending with the engines at idle from the top of descent (TOD) down to the stabilization point, once the instrumental landing system is intercepted. Unfortunately, ATCs have difficulties to accurately predict the vertical profiles and overfly times at certain fixes of such operations. Therefore, larger separation margins are typically required to account for the trajectory uncertainties of aircraft performing CDO. As a result, and to not compromise the runway throughput, CDOs are limited to off-peak hours when the traffic demand is low [4].

A potential approach to enable CDO without a negative impact on the capacity is to separate, sequence, and merge the arrival traffic by assigning to each aircraft the required times of arrival (RTA) at one or several strategic fixes, while ensuring a minimum separation [5] . Then, during the course of the descent, no radar vectors, altitude assignments, or speed adjustment instructions would be given by the ATC, except for unforeseen situations requiring an immediate separation action. As a result, each aircraft would be able to uninterruptedly fly its own optimal descent trajectory.

In the last years, a lot of effort was devoted to the development of advanced onboard planning and guidance concepts capable to accurately comply with these RTA [,,$\underline{7}]$ and to analyze the feasibility of this approach [8,9]. However, most of the previous works did not consider scenarios, in which all the descents subject to RTA are performed with the engines at idle and without deploying speed brake, using only elevator control to adjust the time of arrival (energyneutral trajectories). Therefore, the feasibility and performance of this time-based and environmentally friendly concept of operations are still unknown.

Several studies have dealt with the assignment of RTA (and the quantification of the feasible arrival windows at given metering fixes), when the aircraft is still in cruise, well before the TOD $[10,11]$. Moreover, in [12], a similar assessment was done assuming the RTA has been notified (or updated) once the descent has been already 
initiated. Obviously, larger feasible time windows are found if RTA are notified with enough anticipation, especially if the aircraft is still in cruise and the TOD can be modified. This paper assesses the feasibility of replacing current ATC traffic sequencing and merging procedures in the TMA by assigning RTA to all arriving traffic to safely merge it at a given metering fix. The main novelty of this study is the consideration of scenarios with only energy-neutral CDOs, in which aircraft achieve various times of arrival at the metering fix with only elevator control during the descent. Moreover, in this study, we determine the minimum radius of action (i.e., the distance to the destination where aircraft should receive the RTA) that would be needed to theoretically remove all radar vectoring while still permitting energy-neutral CDOs.

\section{Background}

In this paper, a two-step optimization problem was solved to assess the feasibility of sequencing and merging arrival traffic by means of RTA. During the first step, the trajectory of each aircraft scheduled to land at the selected airport was optimized to determine the earliest, latest, and minimum fuel times of arrival at a certain metering fix. On the base of these arrival times, the optimal RTA that should be assigned to each aircraft, while allowing to safely land all the arriving traffic demand, were found by solving an aircraft scheduling problem [13].

\section{A. Trajectory Optimization Problem Statement}

In this paper, the optimization of the aircraft trajectory is formulated as an optimal control problem. Aircraft dynamics, atmosphere thermodynamic parameters, and path constraints were the problem constraints. A point-mass model was used to describe the aircraft dynamics.

\section{Aircraft Point-Mass Model}

A set of ordinary differential equations can be used to describe the aircraft dynamics. The equations are nonlinear and can describe various physical phenomena (e.g., elasticity [14]). However, structural deformations produce a significant contribution for highaspect-ratio airplanes (mainly gliders). Therefore, for flight dynamics purposes, a six degree-of-freedom (DOF) rigid-body model is usually used [15-17]. This was also done in this study, as Airbus A320 (which was the aircraft under test) aspect ratio is 10.47 [18]. The majority of aircraft have a symmetry plane in terms of geometry and mass, which allows decomposing equations of motion into two sets: longitudinal and lateral-directional. A descent is done in a vertical direction, and so only the longitudinal plane was investigated in this study. To determine the aircraft state for air traffic synchronization purposes, only along path distance, height, velocity, and flight-path angle are necessary. Along path distance and height are used to determine aircraft position on descent route, whereas speed and flight-path angle are used by ATCs to estimate how the position on descent route will change, until receiving the update from the aircraft.

This means that aircraft attitude (pitch angle) will not be used, and the task can be further simplified by adopting a two-dimensional point-mass model with two DOF (velocity and flight-path angle) [19]. This approach is often used when referring to aircraft scheduling problems $[\underline{20}, \underline{21}]$.

Usage of more complex dynamic models, which would exploit additional quantities, system component dynamics (e.g., control system), or impact of the environment would slightly influence the results. However, the calculations would be much longer, and so finding a solution in a reasonable timescale would not be possible, as the simulation of a six DOF linear model of a similar aircraft lasting $60 \mathrm{~s}$ and sampled at $50 \mathrm{~Hz}$ took approximately $2 \mathrm{~s}$ on an Intel Pentium Quad Core laptop running at $2.1 \mathrm{GHz}$ with $4 \mathrm{~GB}$ RAM, in a 64-bit Windows 7 operating system [17]. Therefore, in the study, it was assumed that the aircraft can be treated as a point mass. In addition, wind effects are neglected, as wind velocity can be simply added to the aircraft velocity if required or balanced throughout change in thrust. In the study, vertical equilibrium was assumed, that is, the dynamics of the flight-path angle was not taken into account. This allowed expressing the aircraft dynamics by the following set of nonlinear ordinary differential equations:

$$
\begin{gathered}
m \dot{v}=T_{\text {idle }}-D-m g \sin \gamma \\
\dot{s}=v \cos \gamma \\
\dot{h}=v \sin \gamma
\end{gathered}
$$

in which $(\cdot)$ denotes derivative with respect to time, and $T_{\text {idle }}$ is the idle thrust. Because neither additional thrust nor speed-brake use is permitted throughout the descent, the control vector was composed of the aerodynamic flight-path angle only (i.e., $\boldsymbol{u}=|\gamma|$ ). In the study, it was assumed that the flight-path angle is a direct control, whereas in reality, this is a result of control device usage (e.g., elevator). Incorporation of the relationship between elevator and flight-path angle would require either a three DOF longitudinal model, which would rise the computational effort (due to the reasons mentioned earlier), or a simplified relationship in a linear form, which would decrease the accuracy. Thus, it was decided to use flight-path angle as a control state. The variations in mass $m$ were neglected because the fuel consumption during an idle descent is a very small fraction of the total aircraft mass [3].

It should be noted that Eq. (1) is valid for all the phases comprised between the TOD and the stabilization point (approximately at $1000 \mathrm{ft}$ above the aerodrome level). For the remainder of the descent and for an eventual short cruise phase before the TOD, the aircraft thrust $T$ became a new control and the nominal fuel flow (FF) was computed as a function of thrust $T$, Mach number $M$, and altitude $h$.

All aircraft-related aerodynamic and propulsive functions were developed by using accurate performance data from the manufacturer. Typically, these data are obtained as a result of experimental tests and specified in tabular form. However, optimization solvers based on gradient methods require expressing all the equations of the model with continuous and differentiable functions. Moreover, second-order derivative information is required for Newton-like methods to eliminate converge problems. In this paper, twice differentiability was achieved by approximating the manufacturer data by tensor product cubic B-splines, as suggested in [22].

Regarding the atmosphere, the international standard atmosphere model was considered, which relates the density, pressure, and temperature with the altitude $h$.

\section{Trajectory Optimization Problem Statement}

The optimization of an aircraft trajectory can be formulated as a multiphase constrained optimal control problem, in which it is desired to determine the control history of the aircraft $\boldsymbol{u}(t)$ during a time interval $\left[t_{0}, t_{f}\right]$, in which a given cost function, $J(\boldsymbol{u}(t), \boldsymbol{x}(t), \boldsymbol{p})$ is minimized (or maximized) while satisfying a set of constraint functions, $\boldsymbol{c}(\boldsymbol{u}(t), \boldsymbol{x}(t), \boldsymbol{p}) \leq 0$, and bounds on the state, control, and optional non-time-dependent parameter $\boldsymbol{p}$.

For some simple problems, the solution can be obtained analytically from the optimality necessary and sufficient conditions [23]. However, when highly nonlinear functions or interior point constraints appear in the mathematical model (as in the problem treated herein, in which a time constraint is given at a metering fix located somewhere in the middle of the descent), this is not a straightforward task. In these cases, it is required to use numerical optimization methods.

Numerical methods for solving optimal control problems can be divided into two major classes: indirect methods and direct methods. As the latter group can easily cope with inequality constraints [22], it was used in this study. The direct methods transform the original continuous, and thus, infinite optimal control problem into a (discrete and finite) nonlinear programming (NLP) optimization problem. The time histories of state and control variables were discretized at a set of collocation points, and the system of nonlinear equations given by Eq. (1) was approximated by continuous functions (polynomials). 
The values of the discretized variables, along with some optional nontime-dependent parameters, were the unknowns for the new finite variable problem, which was transformed to an NLP problem and solved by using standard solvers.

The trapezoidal collocation method approach was used in this paper, which is less computationally expensive than other methods based on spectral functions, giving at the same time an accurate result provided that the number of collocation points is large enough $[\underline{12}, \underline{22}]$.

\section{B. Aircraft Scheduling Problem Statement}

Let $\boldsymbol{A}$ be a set that composes all the aircraft scheduled to land at a given airport during a certain interval of time and $\boldsymbol{P}$ a set that contains all possible pairs of aircraft $(p, q)$, with $p, q \in \boldsymbol{A}$ and $p \neq q$. In this paper, it was assumed that well before the TOD, each aircraft computes its own preferred trajectory onboard. This is done on the basis of a cost index, which reflects the relative importance of the cost of time with respect to fuel costs $[21,23]$. From this trajectory, the expected time of arrival (ETA) at a certain metering fix, $\operatorname{ETA}^{(p)}$, can be determined. In addition, the earliest $\mathrm{RTA}_{E}^{(p)}$ and latest $\operatorname{RTA}_{L}^{(p)}$ times of arrival at the metering fix are also extracted from the earliest and latest descent trajectories computed onboard, respectively. Subsequently, these characteristic times of arrival at certain fixes along the route are assumed to be sent to the ground systems via a data link for sequencing and merging purposes, such as the scheduling problem presented in this section.

The goal of the scheduling algorithm is to safely allocate the arrival traffic by assigning an RTA at the metering fix to each aircraft $p$, such that it lies within the feasible earliest-latest window. Moreover, a certain time separation $\Delta t_{\text {sep }}$ is enforced with every preceding and following aircraft $q$, with $p, q \in \boldsymbol{P}$.

The solution of this problem could be feasible or infeasible depending on the complexity of the scenario, which depends on the traffic structure and the required time separation. For the feasible scenarios, there may exist many distinct solutions, in which all the constraints of the problem are satisfied. (The time separation between aircraft is ensured and the RTA assigned to each aircraft fits into its feasible time window.) In this case, the RTA assigned to each aircraft should be as close as possible to the corresponding ETA to minimize the impact of the RTA on the cost of the operation. Therefore, the minimized cost function is

$$
\sum_{p \in A}\left|\operatorname{RTA}^{(p)}-\operatorname{ETA}^{(p)}\right|
$$

subject to the following separation constraints:

$$
\begin{aligned}
& \operatorname{RTA}_{E}^{(p)} \leq \operatorname{RTA}^{(p)} \leq \operatorname{RTA}_{L}^{(p)} \quad \forall p \in \boldsymbol{A} \\
& \left|\operatorname{RTA}^{(p)}-\operatorname{RTA}^{(q)}\right| \geq \Delta t_{\text {sep }} \quad \forall(p, q) \in \boldsymbol{P}
\end{aligned}
$$

\section{Generation and Scheduling of CDO Trajectories}

The minimum fuel and earliest and latest trajectories were computed for each aircraft arriving at the airport subject of study using the trajectory model, which is presented in Sec. III.A. Then, in Sec. III.B, the aircraft landing problem that was solved to schedule the arrival traffic is presented.

\section{A. Trajectory Model}

First, a minimum fuel trajectory was computed while the aircraft is still in the cruise phase, and the ETA at the different fixes of the route were calculated from the minimum fuel trajectory. It has been assumed that the aircraft is following the altitude and speed plans from this initial optimization. Then, when an RTA is issued by the ATC, the earliest and latest trajectories at the metering fix(es) are computed onboard, taking into account the current position of the aircraft. Then, the RTA window together with the ETA is downlinked to the ground system, in charge of the scheduling algorithm.

The minimum fuel trajectories were computed by particularizing the generic optimal control problem described in Sec. II.A.2 with the following cost function:

$$
J=\int_{t_{0}}^{t_{f}} \mathrm{FF}(t) \mathrm{d} t
$$

The earliest and latest trajectories were obtained by minimizing and maximizing, respectively, the time of arrival at the various metering fixes. In this study, only one metering fix was considered, and the intermediate fix (IF) of the instrument approach procedure was chosen. The objective function to optimize is the time of arrival at that fix $t_{\mathrm{IF}}$ :

$$
J=t_{\mathrm{IF}}
$$

To particularize the dynamic constrains point-mass model defined by Eq. (1), was used. The control variables bound were:

$$
\begin{aligned}
& \gamma_{\min } \leq \gamma \leq 0 \\
& T_{\text {idle }} \leq T \leq T_{\max }
\end{aligned}
$$

in which $\gamma_{\min }$ is the minimum flight-path angle, and bounds on thrust are only applicable for the cruise and glide path stabilization phases, since during the descent idle thrust is enforced. In addition, generic path constraints as a function of the state variables have been defined to keep the speed within operational limits:

$$
\begin{aligned}
\mathrm{FAS} & \leq v_{\mathrm{CAS}} \leq \mathrm{VMO} \\
M & \leq \mathrm{MMO}
\end{aligned}
$$

The arrival was split into specific phases and different path and event constraints, in addition to constraints defined by Eqs. (4-7), that may apply in each phase. In Table 1 , various phases and their associated constraints are presented. It should be noted that, in Table $1, t_{0}$ and $t_{f}$ refer, respectively, to the initial and final time of the concerned phase.

The state of the aircraft when receiving the RTA $\boldsymbol{x}_{\mathrm{RTA}}$ fixes the initial condition $\boldsymbol{x}_{0}$ and determines the first phase of the earliest and latest optimization problems. Depending on the active phase when the RTA is received, two cases are possible. In the first case, the aircraft is still in cruise and the TOD can be adjusted to achieve an earlier/later time of arrival maintaining idle thrust during the descent. In the second case, the aircraft has already initiated the descent, and only energy modulation through elevator control is allowed to adjust the time of arrival [].

Table 1 Definition of the flight phases and their constraints

\begin{tabular}{lcc}
\hline \hline Phase & Path constraints & Event constraints \\
\hline $\begin{array}{l}\text { Cruise phase } \\
\text { (if any) }\end{array}$ & $\mathrm{GD} \leq v_{\mathrm{CAS}}(t) ; \dot{M}=0 ;$ & $\boldsymbol{x}_{0}=\boldsymbol{x}_{\mathrm{RTA}}$ \\
$\begin{array}{l}\text { Descent above } \\
\text { FL100 }\end{array}$ & $\mathrm{GD} \leq v_{\mathrm{CAS}}(t)$ & $\boldsymbol{x}_{0}=\boldsymbol{x}_{\mathrm{RTA}}$ \\
$\begin{array}{l}\text { Descent below } \\
\text { FL100 }\end{array}$ & $\mathrm{GD} \leq v_{\mathrm{CAS}}(t) \leq 250 \mathrm{kt}$ & $h\left(t_{0}\right)=10,000 \mathrm{ft}$ \\
$\begin{array}{l}\text { Approach } \\
\text { Leveled }\end{array}$ & $\mathrm{GD} \leq v_{\mathrm{CAS}}(t) \leq 250 \mathrm{ft}$ & $s\left(t_{0}\right)=s_{\mathrm{if}}$ \\
deceleration & $h(t)=2000 \mathrm{ft} ;$ & $v_{\mathrm{CAS}}\left(t_{0}\right)=\mathrm{GD}$ \\
$\begin{array}{l}\text { Deceleration on } \\
\text { glide path }\end{array}$ & $S(t)=-3 \mathrm{deg}$ & $v_{\mathrm{CAS}}\left(t_{0}\right)=S ; s\left(t_{0}\right)=s_{\mathrm{FAP}}(t)$ \\
$\begin{array}{l}\text { Stabilized on } \\
\text { glide path }\end{array}$ & $\gamma(t)=-3 \mathrm{deg} ;$ & $h\left(t_{0}\right)=1000 \mathrm{ft} ;$ \\
\hline \hline
\end{tabular}

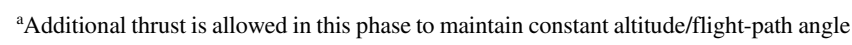
and speed. 
During the cruise phase, if any, the aircraft must keep constant speed and altitude. To provide more flexibility regarding the speed profile when generating the earliest and latest trajectories in cruise, the initial speed is left free between the green $\operatorname{dot}(\mathrm{GD})$ speed, which is the minimum operating speed in clean configuration (corresponding approximately to the best lift-to-drag ratio), and the maximum operational Mach (MMO).

The first descent phase ends at FL100. Below this altitude, ATC procedures typically restrict the calibrated airspeed (CAS) to $250 \mathrm{kt}$. Afterward, the aircraft directs to the IF (located at $s_{\mathrm{IF}}$ ), where the approach phase begins. When reaching GD, the aircraft starts configuring with flaps at constant altitude (still at idle thrust) to intercept the instrumental landing system glide path at the final approach point $s_{\mathrm{FAP}}$ and at the specific speed $S$. For Airbus A320, the aircraft used in this study, the $S$ speed is the target speed when the aircraft is in CONF1 configuration.

When descending on the glide path, the aircraft decelerates at idle thrust and reaches the final approach speed (FAS) at $1000 \mathrm{ft}$, with the gear down and in landing configuration. The last phase ends upon the aircraft reaching $50 \mathrm{ft}$ over the runway threshold at the FAS. It should be noted that nominal configuration transitions are also considered as additional trajectory phases, but not depicted in Table 1 , aiming to keep it simple.

\section{B. Aircraft Scheduling Using Mixed-Integer Linear Programming}

The generic aircraft scheduling problem is a discontinuous NLP optimization problem because the absolute value functions appearing in Eqs. (2) and (3) lead to discontinuous derivatives of the cost function. The problem can be linearized through reformulation of the absolute value expressions appearing in the cost function and constraints. The resulting linear problem is easier to handle from a numerical point of view.

On the one hand, the separation constraint defined by Eq. (3) can be reformulated by means of the following two classical disjunctive constraints [24]:

$$
\begin{aligned}
& \operatorname{RTA}_{E}^{(p)}+\Delta t_{\text {sep }} \leq \operatorname{RTA}^{(q)}+L^{(p, q)} B^{(q, p)} \forall(p, q) \in \boldsymbol{P}, \quad q<p \\
& \operatorname{RTA}_{E}^{(p)}+\Delta t_{\text {sep }} \leq \operatorname{RTA}^{(q)}+L^{(p, q)}\left(1-B^{(p, q)}\right) \quad \forall(p, q) \in \boldsymbol{P}, \quad p<q
\end{aligned}
$$

in which $B^{(p, q)}$ for $p<q$ is a binary variable that takes a value of 1 if aircraft $p$ arrives before aircraft $q$ and 0 otherwise, and $L^{(p, q)}$ is a relatively large scalar [24].

To avoid problems with numerical stability, the value of $L^{(p, q)}$ should not be chosen too large. The minimum value of $L^{(p, q)}$, high enough to satisfy Eq. (ㅇ), was obtained from

$$
L^{(p, q)}=\max \left\{\operatorname{RTA}_{L}^{(p)}-\operatorname{RTA}_{E}^{(q)}, \operatorname{RTA}_{L}^{(q)}-\operatorname{RTA}_{E}^{(p)}\right\} \forall(p, q) \in \boldsymbol{P}, \quad q<p
$$

A common approach to linearize the cost function is to express the term inside the absolute value as the difference of two artificial and positive variables [25], namely, $\Delta t_{+}^{(p)}$ and $\Delta t_{-}^{(p)}$, and rewrite the absolute value function in Eq. (2) as the sum of these two variables:

$$
\left|\operatorname{RTA}^{(p)}-\operatorname{ETA}^{(p)}\right|=\Delta t_{+}^{(p)}+\Delta t_{-}^{(p)} \quad \forall p \in \boldsymbol{A}
$$

By using Eqs. (ㅁ-10), the discontinuous NLP aircraft scheduling problem given by Eqs. (2) and (3) can be reformulated. In the resulting mixed-integer linear programming (MILP) problem, some of the variables are restricted to be integers, while the objective function and the constraints are linear. For the reformulated problem, the following cost function is minimized:

$$
\sum_{p \in A}\left(\Delta t_{+}^{(p)}+\Delta t_{-}^{(p)}\right)
$$

subject to the following constraints:

$$
\begin{aligned}
& \operatorname{RTA}_{E}^{(p)} \leq \operatorname{RTA}^{(p)} \leq \operatorname{RTA}_{L}^{(p)} \forall p \in \boldsymbol{A} \\
& \operatorname{RTA}^{(p)}+\Delta t_{\text {sep }} \leq \operatorname{RTA}^{(q)}+L^{(p, q)} B^{(q, p)} \quad \forall(p, q) \in \boldsymbol{P}, \quad q<p \\
& \operatorname{RTA}^{(p)}+\Delta t_{\text {sep }} \leq \operatorname{RTA}^{(q)}+L^{(p, q)}\left(1-B^{(p, q)}\right) \quad \forall(p, q) \in \boldsymbol{P}, \quad p<q \\
& B^{(p, q)} \in\{0,1\} \quad \forall(p, q) \in \boldsymbol{P}, \quad p<q \\
& \operatorname{RTA}^{(p)}-\operatorname{ETA}^{(p)}=\Delta t_{+}^{(p)}+\Delta t_{-}^{(p)} \quad \forall p \in \boldsymbol{A} \\
& \Delta t_{+}^{(p)}, \quad \Delta t_{-}^{(p)} \geq 0 \quad \forall p \in \boldsymbol{A}
\end{aligned}
$$

This formulation of the scheduling problem assumes that the time windows for all the pairs of aircraft could overlap. However, in many practical scenarios, this generic formulation is not efficient, because there may exist redundant variables and constraints that unnecessary increase the complexity of the problem.

A more efficient algorithm can be developed by classifying the pairs of aircraft into three different sets [24]: the first set $\boldsymbol{P}_{\text {sep }}$ contains pairs of aircraft $(p, q)$ with guaranteed separation, that is, when $\mathrm{RTA}_{L}^{(p)}+\Delta t_{\text {sep }} \leq \mathrm{RTA}_{E}^{(q)}$ or RTA ${ }_{L}^{(q)}+\Delta t_{\text {sep }} \leq \mathrm{RTA}_{E}^{(p)}$ is fulfilled. For these pairs of aircraft, separation is inherently satisfied regardless of the assigned RTA. The second set, $\boldsymbol{P}_{\text {nonsep }}$, is composed of the pairs of aircraft $(p, q)$ with disjoint time windows without guaranteed separation, that is, in which RTA ${ }_{L}^{(p)} \leq \mathrm{RTA}_{E}^{(q)}$ and $\mathrm{RTA}_{L}^{(q)} \leq \mathrm{RTA}_{E}^{(p)}$, but when $\mathrm{RTA}_{L}^{(p)}+\Delta t_{\text {sep }}>\mathrm{RTA}_{E}^{(q)}$ or $\mathrm{RTA}_{L}^{(q)}+\Delta t_{\text {sep }}>\mathrm{RTA}_{E}^{(p)}$ applies. The last set, $\boldsymbol{P}_{\text {overlap }}$, contains the pairs of aircraft $(p, q)$ with overlapping time windows. Considering these sets, the following constraints can be added to Eq. (12), aiming to reduce the computational burden:

$$
\begin{aligned}
& \forall(p, q) \in \boldsymbol{P}_{\text {sep }} \cup \boldsymbol{P}_{\text {nonsep }}, \quad \operatorname{RTA}_{L}^{(p)}<\operatorname{RTA}_{E}^{(q)}: B^{(p, q)}=1 \\
& \forall(p, q) \in \boldsymbol{P}_{\text {sep }} \cup \boldsymbol{P}_{\text {nonsep }}, \quad \operatorname{RTA}_{L}^{(q)}<\operatorname{RTA}_{E}^{(p)}: B^{(q, p)}=1 \\
& \forall(p, q) \in \boldsymbol{P}_{\text {nonsep }}, \quad \operatorname{RTA}_{L}^{(p)}<\operatorname{RTA}_{E}^{(q)}: \operatorname{RTA}^{(p)}+\Delta t_{\text {sep }} \leq \operatorname{RTA}^{(q)} \\
& \forall(p, q) \in \boldsymbol{P}_{\text {nonsep }}, \quad \operatorname{RTA}_{L}^{(q)}<\operatorname{RTA}_{E}^{(p)}: \operatorname{RTA}^{(q)}+\Delta t_{\text {sep }} \leq \mathrm{RTA}^{(p)}
\end{aligned}
$$

The first two constraints of Eq. (13) enforce the variables $B^{(p, q)}$ to be either 0 or 1 in the case of disjoint time windows. The latter two constraints are simple precedence constraints.

\section{Results}

The assessment was performed for Barcelona-El Prat Airport (Spain) using historic flight traffic data between 1 January and 31 July 2016. Days when the West Configuration was selected for aircraft arrival with various traffic intensities were chosen from this time period. The data were obtained from the EUROCONTROL's Demand Data Repository [26], which contains information about the trajectories flown every day. In this paper, three days, representing low, moderate, and high arrival traffic densities, are presented.

\section{A. Experimental Setup}

Barcelona-El Prat Airport uses three runways with five directions for landing [27]. The West Configuration is the most typically used, with runways $25 \mathrm{~L}$ and $25 \mathrm{R}$ for takeoff and landing, respectively. This configuration uses 11 possible standard terminal arrival routes (STARs) with their corresponding entry points [28], as shown in Fig. 1. These STARs coincide into four possible initial approach fixes that finally merge all traffic into a single IF. (TEBLA is the IF for the West Configuration.)

To obtain the Aeronautical Information Regulation and Control files [29] that contain 28 days of traffic data from the Demand Data Repository, the NEST software, provided by EUROCONTROL, was used. The software was used to create custom traffic flow to extract the arriving traffic for the airport of interest. In the obtained data, all flight segments for each flight were described by 20 fields, 


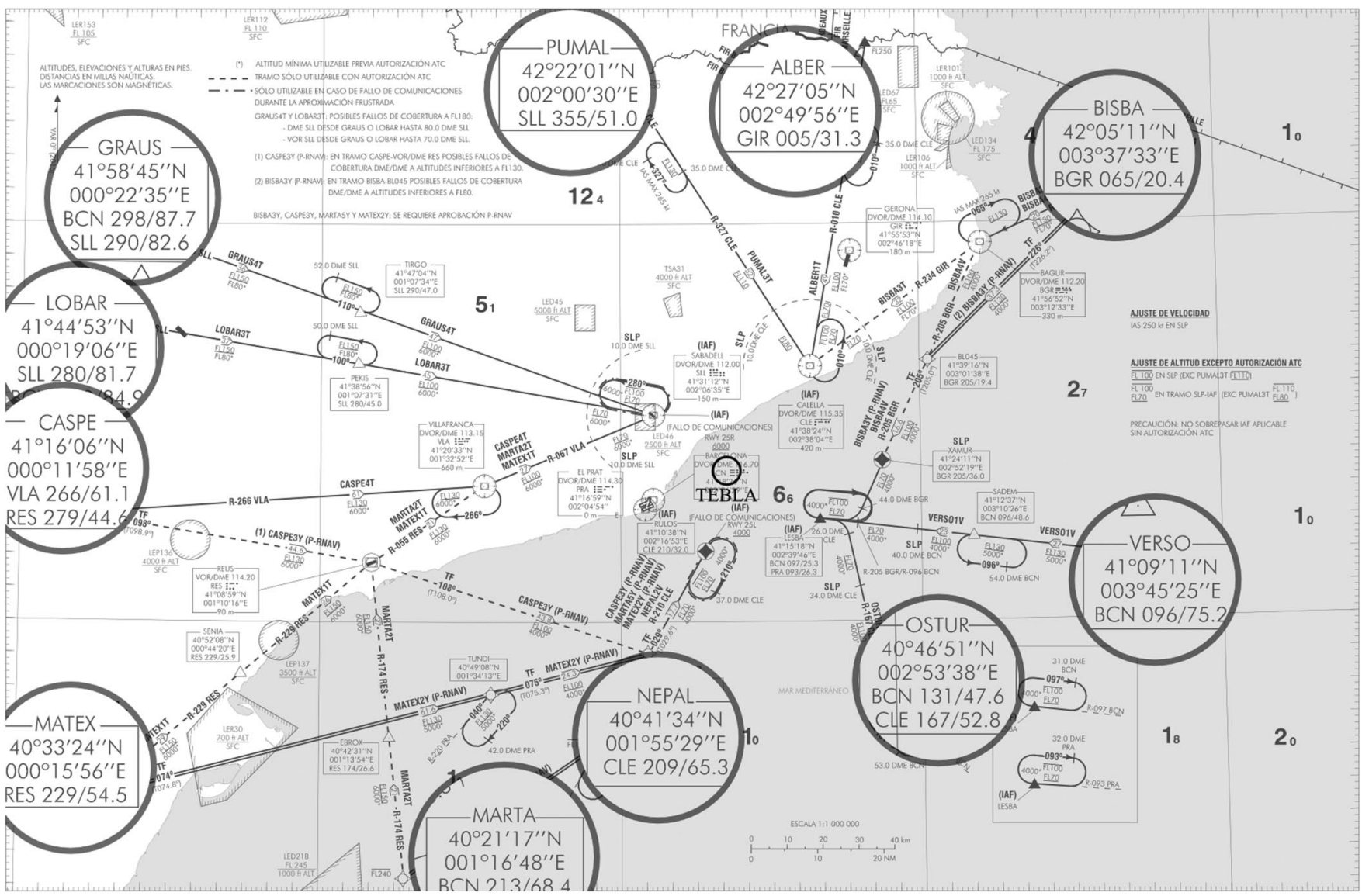

Fig. 1 Barcelona-El Prat STARs for the West Configuration [28].

which include four-dimensional (4-D) trajectory data (latitude, longitude, altitude, and time) for the segment boundaries, as well as aircraft and flight identity codes. The structure of the data file is presented in Table 2 [30].

In the obtained data, two types of trajectories were stored: planned trajectory, which is equal to the last filled flight plan, and actual trajectory, which is the planned trajectory updated with radar data. The planned and actual trajectories are presented in Fig. 2 with black and gray lines, respectively. It can be seen in Fig. $2 \mathrm{a}$ that the difference between the planned and actual trajectories may be noticeable in certain cases. Figure $2 \mathrm{~b}$ shows a particular flight, in which a change in airport landing configuration occurred.

In this study, actual trajectories were used, as they are closer to actual data. (Planned trajectories have less data points, especially in the descent.) Furthermore, actual trajectories preserve true separation values between the aircraft, whereas planned trajectories are only computed from flight plans submitted by the aircraft operators.

The completeness of the data files was checked by running data parsing script with no errors. From these data, the aircraft times of arrival to navigation points of interest were obtained (i.e., the times of arrival at TEBLA, STAR entry points, and other navigation points that were at a certain distance from the runway threshold). While the first two were directly read from the parsed data, the latter was obtained by finding the appropriate flight segment and calculating the time with the assumption that the aircraft flew at a constant ground speed in that segment. The correctness of the data was checked by assessing whether the obtained values are typical for given flight segments. The presence of appropriate fixes and distances to the runway from those fixes were checked, as well as the time used to reach the runway from those fixes. The presence of singular points (i.e., peaks or dips) was treated as bad data, and flights for which they occurred were deleted from the arrival set. The rejected data mean for 178 days was $9.56 \%$ with a corresponding standard deviation of
Table 2 Flight data file structure

\begin{tabular}{|c|c|c|c|c|}
\hline Number & Field & Type & Size & Comment \\
\hline 1 & Segment identifier & Char & $8-10$ & $\begin{array}{l}\text { First point name " } \\
\text { last point }\end{array}$ \\
\hline 2 & Origin of flight & Char & 4 & ICAO code \\
\hline 3 & $\begin{array}{l}\text { Destination of } \\
\text { flight }\end{array}$ & Char & 4 & ICAO code \\
\hline 4 & Aircraft type & Char & 4 & ICAO code \\
\hline 5 & $\begin{array}{c}\text { Time begin } \\
\text { segment }\end{array}$ & Num & 6 & HHMMSS \\
\hline 6 & Time end segment & Num & 6 & HHMMSS \\
\hline 7 & $\begin{array}{l}\text { Flight level begin } \\
\text { segment }\end{array}$ & Num & $1-3$ & -- \\
\hline 8 & $\begin{array}{l}\text { Flight level end } \\
\text { segment }\end{array}$ & Num & $1-3$ & -- \\
\hline 9 & Status & Char & 1 & $\begin{array}{c}0=\text { climb } ; 1=\text { descent } \\
2=\text { cruise }\end{array}$ \\
\hline 10 & Call sign & Char & Var & -- \\
\hline 11 & $\begin{array}{c}\text { Date begin } \\
\text { segment }\end{array}$ & Num & 6 & YYMMDD \\
\hline 12 & Date end segment & Num & 6 & YYMMDD \\
\hline 13 & $\begin{array}{l}\text { Latitude begin } \\
\text { segment }\end{array}$ & Float & Var & In minute decimals \\
\hline 14 & $\begin{array}{l}\text { Longitude begin } \\
\text { segment }\end{array}$ & Float & Var & In minute decimals \\
\hline 15 & $\begin{array}{l}\text { Latitude end } \\
\text { segment }\end{array}$ & Float & Var & In minute decimals \\
\hline 16 & $\begin{array}{l}\text { Longitude end } \\
\text { segment }\end{array}$ & Float & Var & In minute decimals \\
\hline 17 & Flight identifier & Num & 9 & Unique for every flight \\
\hline 18 & Sequence & Num & Var & -- \\
\hline 19 & Segment length & Float & Var & In nautical miles \\
\hline 20 & $\begin{array}{l}\text { Segment parity/ } \\
\text { color }\end{array}$ & Num & 1 & -- \\
\hline
\end{tabular}

$\mathrm{ICAO}=$ International Civil Aviation Organization, $\mathrm{Char}=$ character, Num $=$ numeric Float $=$ floating point number, Var $=$ variable length, $\mathrm{HHMMSS}=2$-digit hour, minute and second format, and YYMMDD = 2-digit year,month and day format. 


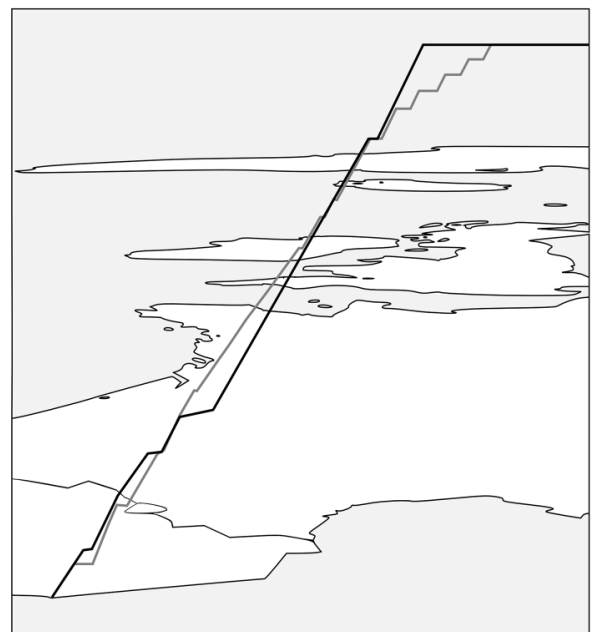

a) No change in airport landing configuration occurred

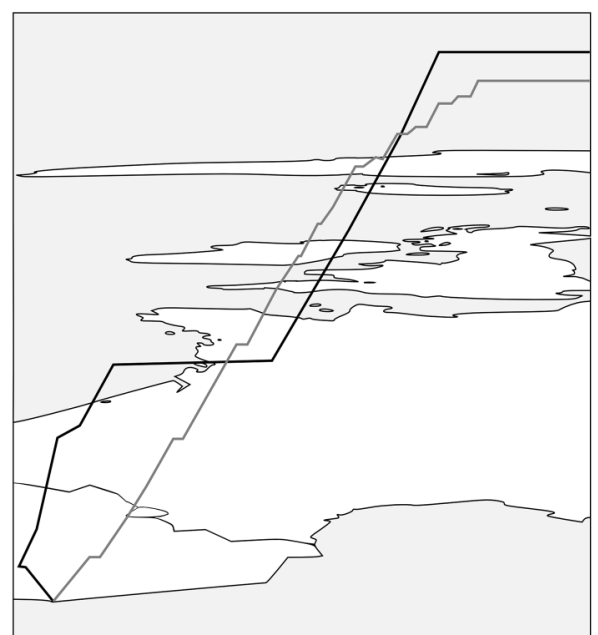

b) Change in landing configuration occurred

Fig. 2 Planned and actual trajectories visualized in NEST.

$3.81 \%$. As mentioned, three days, representing low, moderate, and high arrival traffic densities, are presented in this paper. For each day, five different cases were analyzed. In the first case, it was assumed that RTA are notified when aircraft reach the entry point of their corresponding STAR. For the remaining cases, RTA were assumed to be given at a certain distance from the runway threshold: 100, 150, 200 , and $250 \mathrm{n}$ mile.

It was observed that, during the analyzed period of time, Airbus A320 was the most often arriving airplane with a $42.6 \%$ share of all arriving aircraft. Moreover, the initial assessment showed that $83.3 \%$ of the aircraft arriving at Barcelona-El Prat are comparable in terms of mass, dimensions, and performance (i.e., Boeing 737). Therefore, Airbus A320 was selected as the representative aircraft for this study, using its performance model to generate the fuel-optimal, earliest, and latest trajectories for each flight.

The minimum flight-path angle was set to $-15 \mathrm{deg}$. The distance from TEBLA to the runway threshold was fixed at $11 \mathrm{n}$ mile, according to the approach chart. For each scenario, the GD speed was computed [31]. The characteristic speeds, $S$ and FAS, were computed based on the stall speed, which is a function of both aircraft mass and configuration. Finally, MMO and maximum operational $v_{\mathrm{CAS}}$ (VMO) were set to 0.80 and $340 \mathrm{kt}$, respectively.

As described earlier, for each scenario and case study, the optimal trajectories in terms of fuel consumption were computed for each aircraft arriving at the airport. In addition, the earliest and latest energy-neutral times of arrival at TEBLA were computed to estimate the energy-neutral RTA window. The optimal trajectories were obtained using CONOPT as NLP solver, which is bundled into the GAMS software suite. CONOPT was chosen as the NLP solver, as it was designed to cope with various NLP problems, also with the ones with a small number of DOF. This solver was used many times in previous studies for trajectory optimization during descent $[20,21]$. The design variables were flight-path angle, thrust, velocity, and Mach number. The optimization was stopped if the relative change in the cost function was $3 \cdot 10^{-12}$ for 20 consecutive well-behaved iterations or iterations with slow convergence. Changes smaller than $3 \cdot 10^{-13}$ were ignored. To determine good search direction, a scaling factor, which was an absolute of a product of a variable and its derivative, was used. The scale factors were projected into the range $\left\langle 1,10^{9}\right\rangle$. To avoid problems with assigning a low weight to model aspects that are significant, derivatives values were bounded to $\left\langle 10^{-5}, 10^{10}\right\rangle$. To avoid scaling problems, the upper bounds were also defined for variables and all function values. This limit was set to $4 \cdot 10^{-10}$ [32].

For all of the cases and scenarios, the time separation was set to $90 \mathrm{~s}$. This value was found to be reasonable, as it compares with current distance separation safety requirements [33].
For every scheduling problem, a relaxation process, which relieves processing unit by dividing the overall problem on smaller subproblems, was performed. Every scheduling problem was divided into subproblems with subsets of flights from a given set. After the partitioning, in each subset there were no flights with required separation within that subset. The optimized sequences of arriving aircraft were obtained by solving MILP scheduling problems using the CPLEX solver, which is designed to solve large-scale mixed-integer linear and quadratic programming problems. The MILP problem was solved with the use of a branch-and-bound algorithm. The inputs were the earliest and latest RTA, minimum-fuel trajectory RTA, and precedence binary values for each aircraft. A dual simplex algorithm was used to solve MILP subproblems. Stop criterion was expressed by a relative change of the cost function between the iterations (convergence tolerance), or this value divided by the range of the variable (optimality tolerance). The convergence tolerance for linear and quadratic programming problems was set to $10^{-8}$ and the optimality tolerance to $10^{-6}$ [34] .

\section{B. Earliest and Latest Trajectories}

In Fig. 3 , the minimum fuel trajectory for an aircraft located at $200 \mathrm{n}$ mile from the runway threshold is shown. The black solid line denotes altitude, the black dashed line the CAS, the gray dashed line the true airspeed, and the gray solid line the Mach number. As it can be seen in the plot, the altitude and speed profile is in accordance with [35]: the aircraft starts descent early, with small variations in

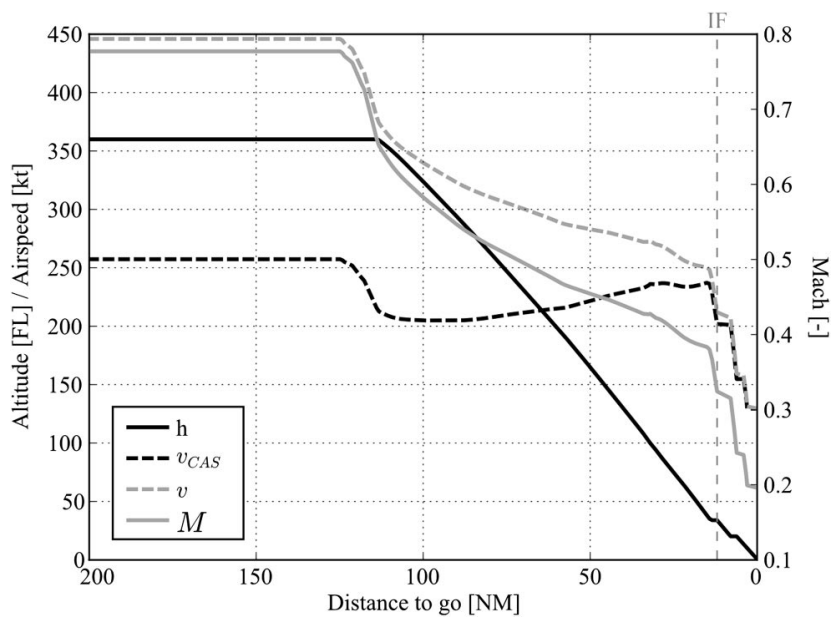

Fig. 3 Minimum fuel trajectory. 


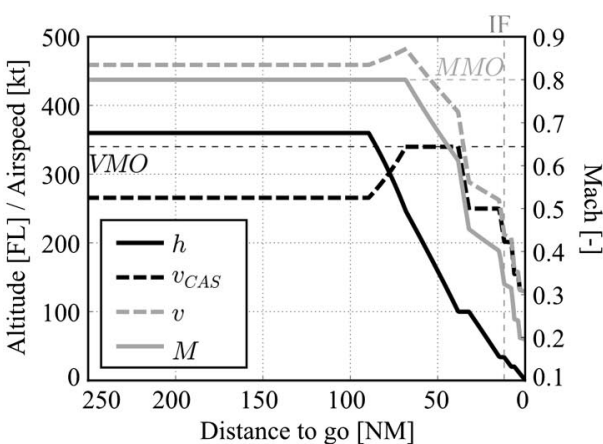

a) $250 \mathrm{n}$ mile (earliest)

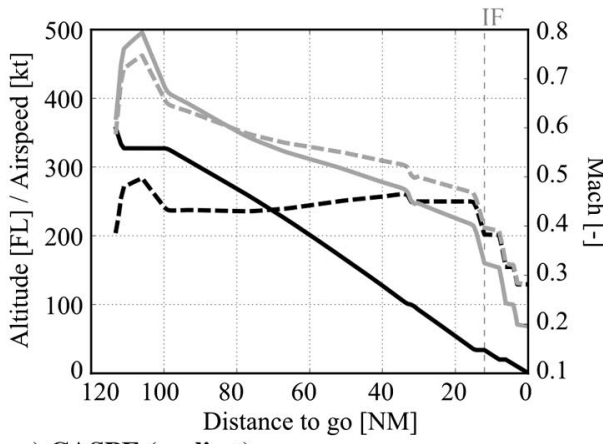

c) CASPE (earliest)

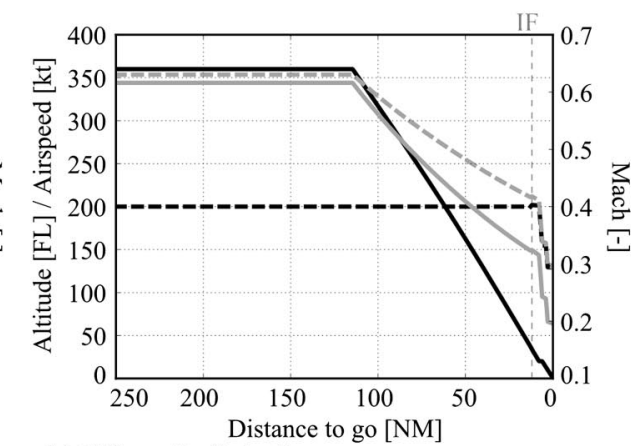

b) $250 \mathrm{n}$ mile (latest)

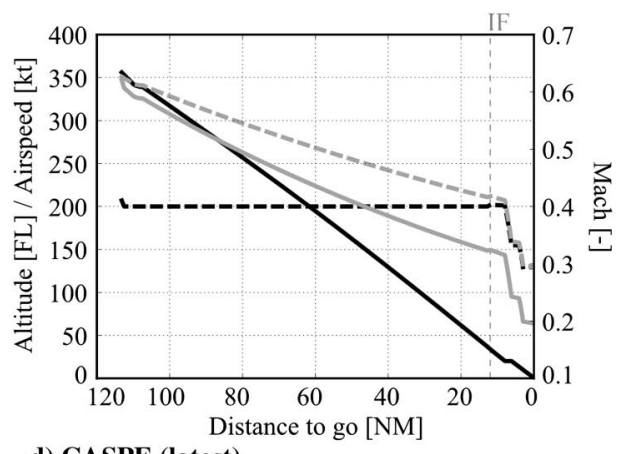

d) CASPE (latest)

Fig. 4 Earliest and latest trajectories.

flight-path angle and with a speed that slowly increases as the aircraft descends.

As commented before, in the study, it was assumed that all aircraft were flying this trajectory before starting the RTA negotiation process. At this moment, the earliest and latest energy-neutral trajectories at the metering fix starting at the current aircraft position are computed and downlinked the feasible RTA window to the ground system.

In Fig. 4, the earliest and latest trajectories for two different RTA negotiation points are presented. Figures $\underline{4 a}$ and $\underline{4 b}$ show the case, in which the RTA was negotiated at $250 \overline{\mathrm{n}}$ mile from the runway threshold, whereas Figs. 4c and 4d correspond to the case, in which the RTA was negotiated at CASPE, the entry point of one of the STARs shown in Fig. 1.

For the case in which the RTA was notified at $250 \mathrm{n}$ mile from the runway threshold and the earliest time trajectory (Fig. 4a), the aircraft was flying in the cruise phase at MMO as long as possible. The first phase of the energy-neutral descent, performed at MMO, starts at the TOD and ends when the CAS reaches VMO at the crossover altitude. Then, the aircraft flies at VMO down to FL100, where a deceleration at constant altitude (still at idle thrust) is performed to comply with the speed constraint enforced at that altitude. Thereafter, the maximum allowed speed below FL100 (250 kt) is maintained until few nautical miles before TEBLA, where another deceleration at constant altitude and idle thrust is performed to reach this fix at the GD speed. On the other hand, for the latest time trajectory (Fig. 4b), both cruise and descent phases are performed at the lowest permitted speed (GD). It is interesting to note that the TOD of the latest time trajectory is located $20 \mathrm{n}$ mile behind that of the earliest time case (110 $\mathrm{n}$ mile for the latest time; $90 \mathrm{n}$ mile for the earliest time).

If the RTA is received at CASPE notification point, the descent has been already initiated. In this case, and because the use of throttle and speed brakes is restricted all along the descent aiming to reduce the environmental impact, only energy modulation through elevator control is permitted to adjust the time of arrival. The RTA negotiation is initiated at $113 \mathrm{n}$ mile from the runway threshold, where the more fuel-efficient trajectory was flying close to the minimum speed at FL350. For the earliest time trajectory, the aim is to accelerate as much as possible, given the available energy. The best practice is to first exchange potential energy (altitude) by kinetic energy (speed) at the maximum gradient, and then to keep the maximum speed, such that the associated drag does not make the energy level drop below that required at TEBLA. On the other hand, the latest time trajectory (Fig. 4d) is performed at GD until reaching TEBLA.

In Fig. 5, the earliest, latest, and fuel-optimal times of arrival at TEBLA for the different RTA negotiation points are shown with triangle, star, and dot markers, respectively. Solid lines represent the feasible time windows. The remaining distance to go is also shown in the right vertical axis for the sake of completeness.

As expected, the farthest from the runway threshold the RTA is negotiated, the wider the feasible RTA window is. According to Fig. 3, the TOD of the fuel-efficient trajectory (before enforcing an RTA $\overline{)}$ is located at approximately $115 \mathrm{n}$ mile from the runway threshold. This fact implies that, for RTA negotiation points closer to the runway threshold, the aircraft has already initiated the descent when the RTA is received, and only energy modulation through elevator control is allowed to adjust the time of arrival at the metering fix.

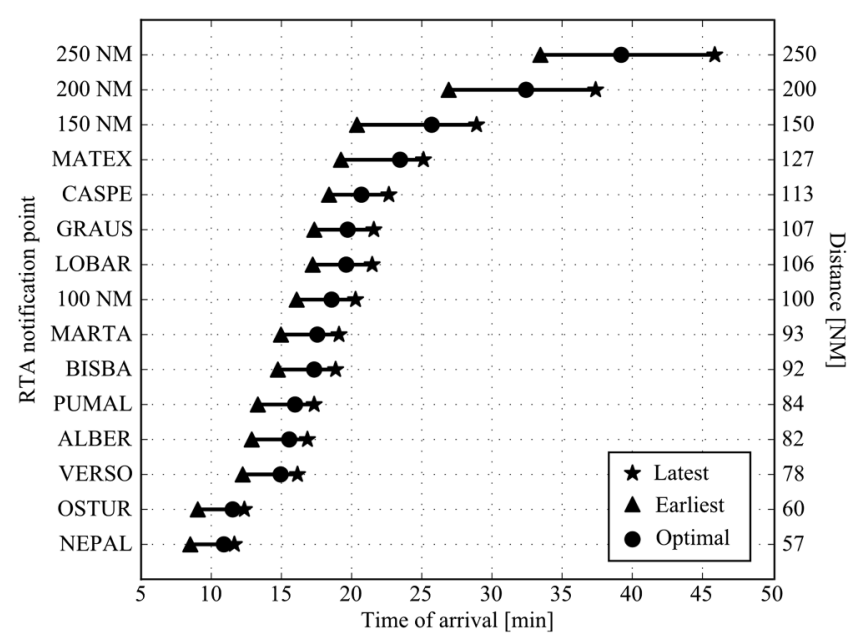

Fig. 5 Earliest, latest, and fuel-efficient times of arrival at TEBLA. 
For the RTA negotiation points with distances longer than $115 \mathrm{n}$ mile, the feasible RTA window rapidly increases due to the fact that no restrictions are enforced for the duration of the cruise phase, provided that the whole descent is performed at idle thrust and without speed-brake use.

\section{Concept Feasibility Assessment}

In this study, five distances where RTA are assigned were analyzed, all corresponding to different RTA negotiation points: the currently published STAR entry points (see Fig. 1) and four hypothetical cases with all entry points fixed at 100, 150, 200, and $250 \mathrm{n}$ mile from the runway threshold. The distance of $100 \mathrm{n}$ mile was chosen as the value close to the average length of the STAR routes, the distance of $250 \mathrm{n}$ mile was chosen as the value considered to be the radius of future TMAs [36], and distance values between that two were calculated to be equally spaced.

Each case corresponded to a different scheduling problem, because the aircraft distribution as well as the RTA windows and ETAs were different. These five cases are shortly denoted as STAR, D100, D150, D200, and D250, respectively. The analysis performed for each case resulted in finding the optimal solution for the whole problem or for a part of the subproblems. Three sample days that represent different arrival traffic intensities are shown herein to present various outcomes of the scheduling process.

In the study, 9 January 2016 was selected as the representative of a day with low arrival traffic. In that day, 201 arrivals took place between 0600 and $2200 \mathrm{hrs}$ through TEBLA. There existed pairs of flights for which separation was smaller than the minimum required in this study. For the five analyzed cases, 54, 53, 28, 19, and 13 subproblems were created, respectively, and all of them were scheduled successfully. In each case, each pair of aircraft was able to maintain a required separation value while performing energyneutral CDOs.

The histograms of the time separations at TEBLA are shown in Fig. 6. On the top left plot, actual time separations between aircraft at the IF, gathered from EUROCONTROL's traffic data, are shown. The remaining histograms correspond to the five different analyzed cases. The results show a normalized number of aircraft that was calculated as the number of flights in a given bin divided by the total number of flights in that day. The arrival times at TEBLA for consecutive aircraft are presented in Fig. 7. In the plot, each aircraft is marked by the black plus sign.

From Fig. 6, it can be observed that approximately $40 \%$ of the flights were performed with $90 \mathrm{~s}$ separation. This value was

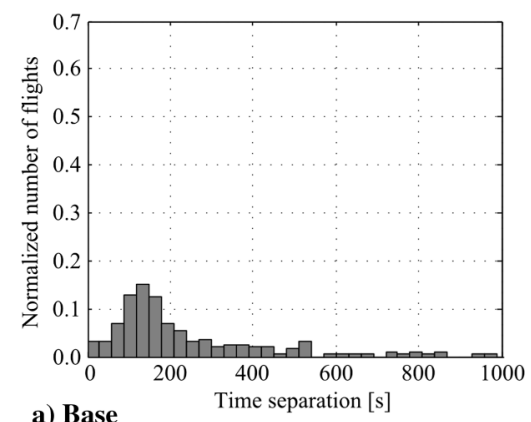

a) Base

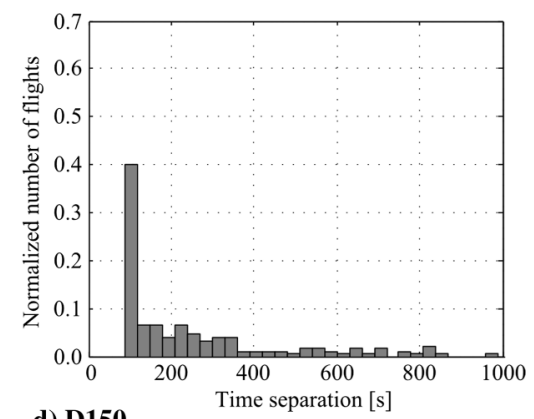

d) D150

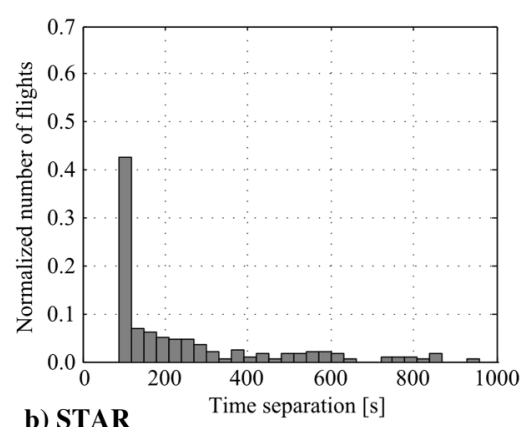

b) STAR

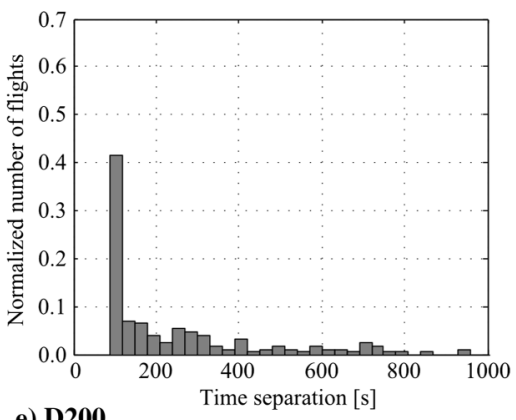

e) D200

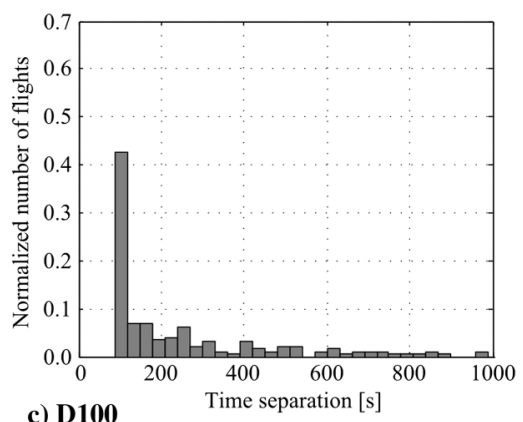

c) $\mathrm{D} 100$

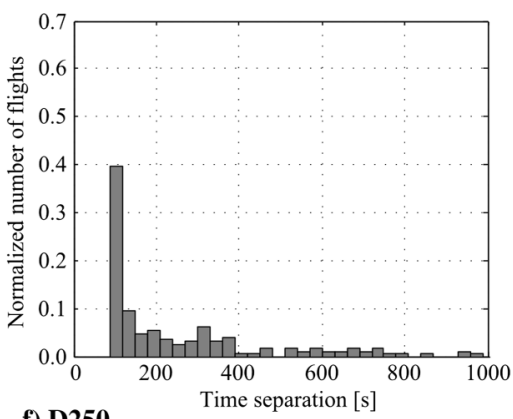

Fig. 6 Time separations at TEBLA on 9 January 2016.

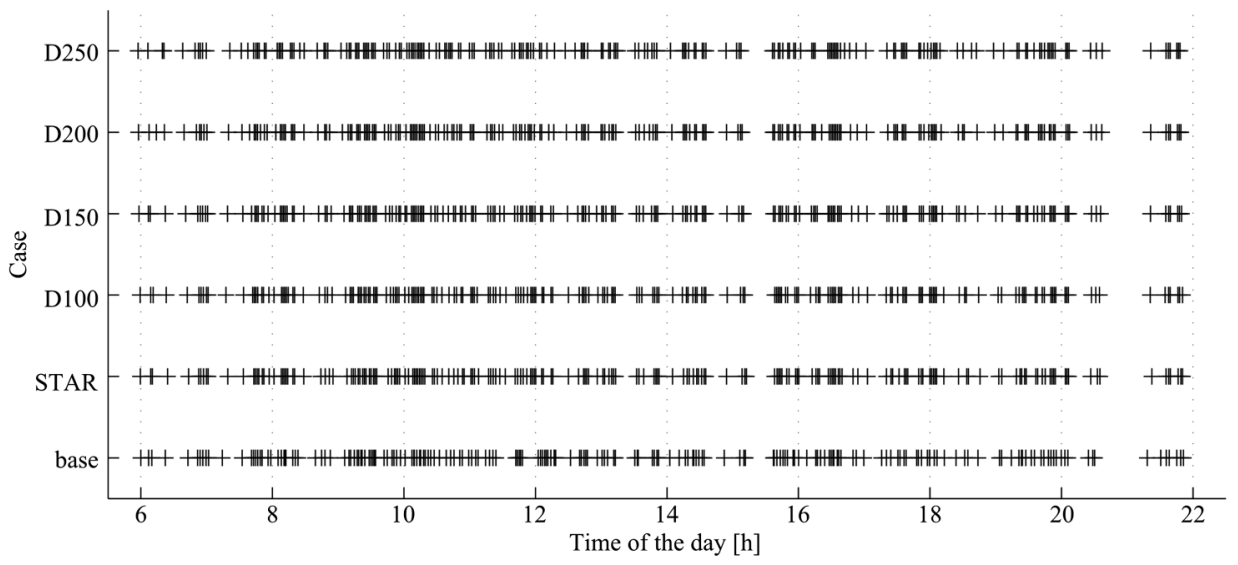

Fig. 7 Times of arrival at TEBLA on 9 January 2016. 
independent of the distance. Moreover, for the analyzed cases, this was the minimal possible separation value-no flights were scheduled with a lower separation value. In reality (base case), the highest number of flights also had a separation of $90 \mathrm{~s}$. For all distances, it was observed that less number of flights were scheduled in the way that their separation was higher. The variations between results are mainly due to differences in descent trajectories for given cases.

By analyzing the results presented in Fig. 6 , it can be concluded that the presented method can be applicable to low-traffic scenarios without a need for extending the TMA radius, as all traffic was successfully synchronized for the STAR case (Fig. 6b).

The feasibility and performance of this sequencing and merging method were also analyzed for a day with moderate arrival traffic: 15 March 2016. On this day, 272 arrivals took place between 0600 and 2200 hrs using the West Configuration. In Fig. $\underline{8}$, the separation times between pairs of aircraft at TEBLA are shown. The arrival times at TEBLA for consecutive aircraft are presented in Fig. 9.
According to Fig. $\underline{8}$, the historical flight data contained pairs of aircraft without a feasible value of separation. For the analyzed cases, $51,45,17,13$, and 10 subproblems were created, respectively. In the cases of STAR and D100, there existed around 30 min sequence of flights that were unable to be scheduled. This time interval is marked in Fig. 9 as a gray box. For the other cases, all aircraft were scheduled successfully.

For the feasible cases of scenario presented in Fig. 8, it was observed that the results are analogical as for the low-intensity scenario (Fig. 6). Approximately $50 \%$ of flights were performed with minimal possible separation (90 s). Like in the low-intensity scenario, for the base case, the highest number of flights had a separation of $90 \mathrm{~s}$. Similarly, as for the analyzed feasible test cases (D150, D200, and D250), for the base case, the number of flights with a minimal possible separation also increased (to approximately 20\%). For all distances, it was observed that less flights are performed with higher separations. This decrease was faster than in the low-intensity scenario. For a greater set of separation values, no flights were scheduled.
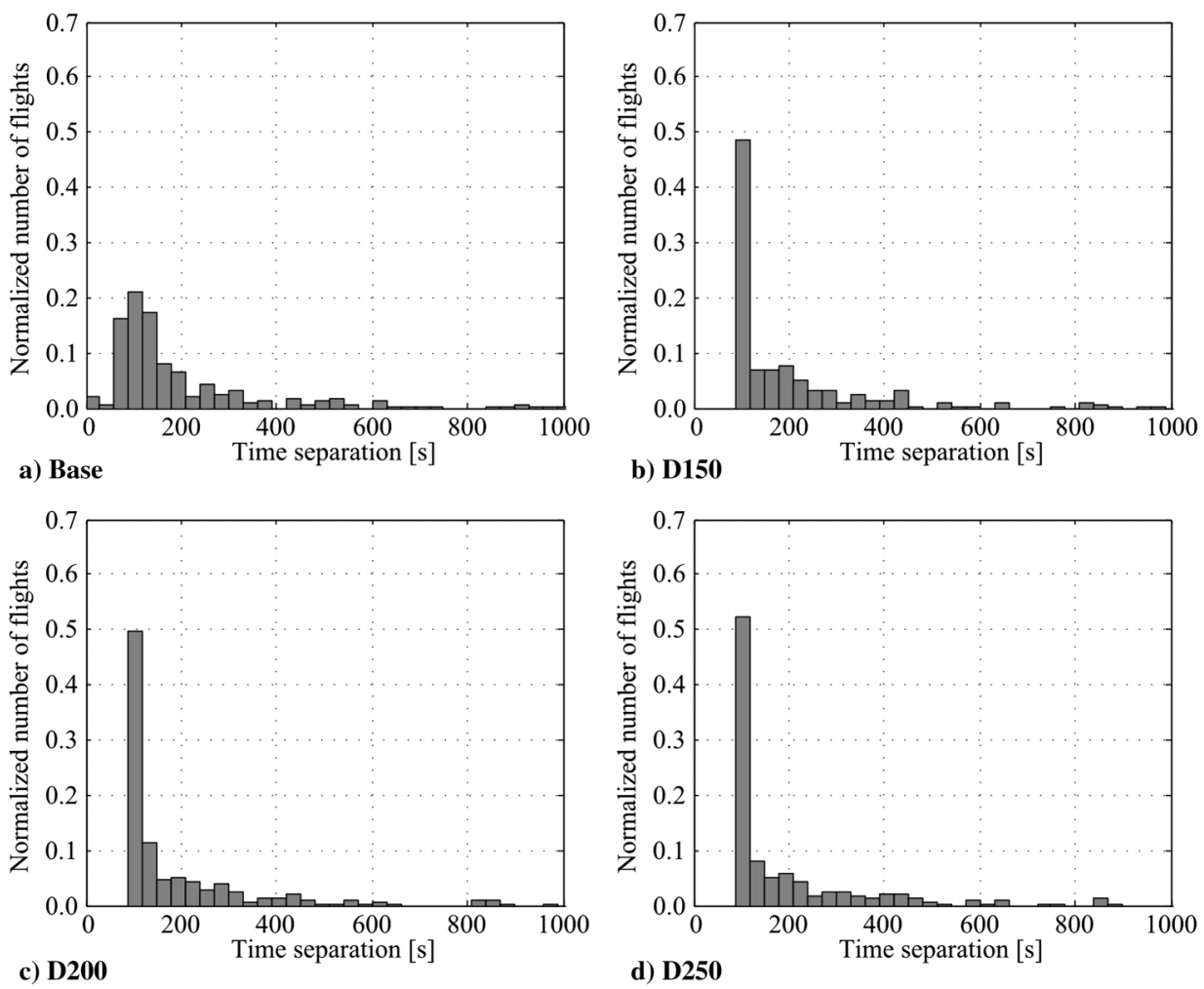

Fig. 8 Time separations at TEBLA on 15 March 2016.

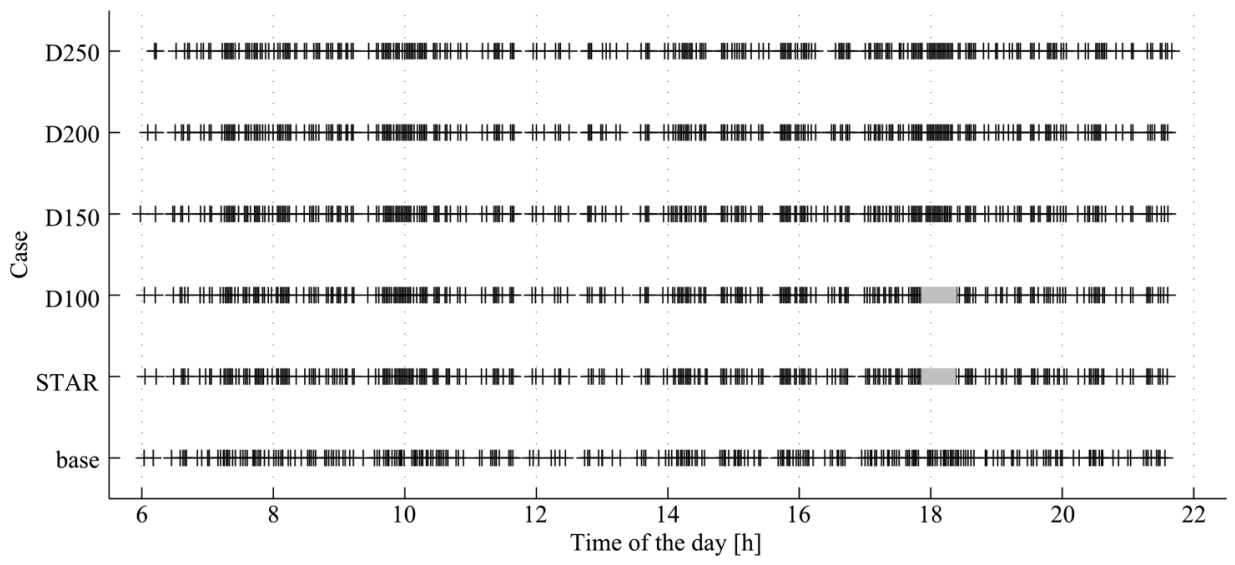

Fig. 9 Times of arrival at TEBLA on 15 March 2016. 


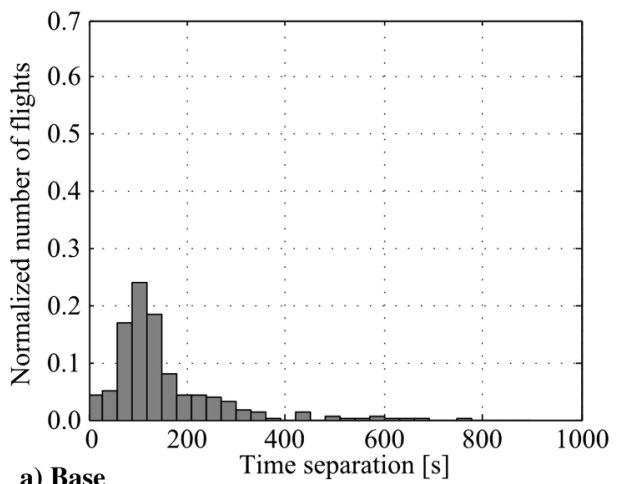

a) Base

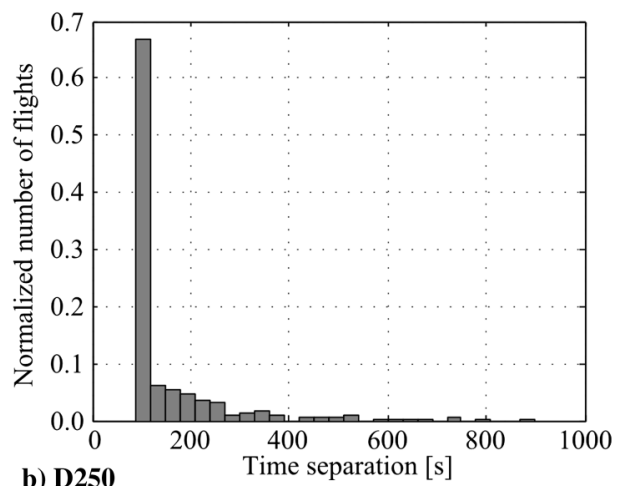

b) D250

Fig. 10 Time separations at TEBLA on 26 April 2016.

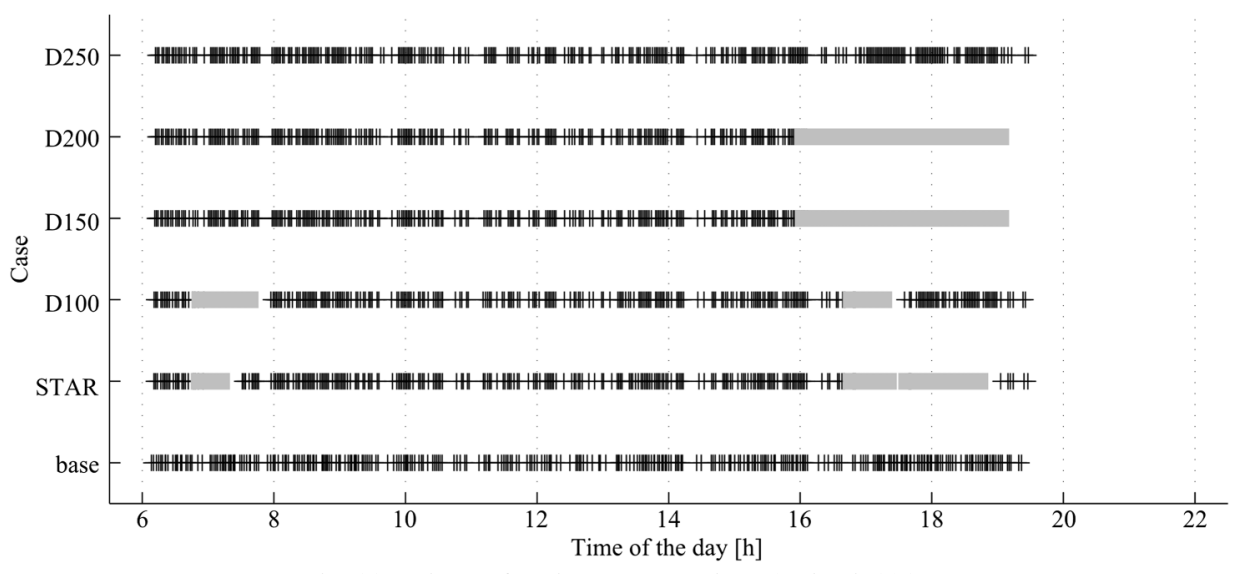

Fig. 11 Times of arrival at TEBLA on 26 April 2016.

The results for the day with moderate traffic intensity show the effect of extending TMA, as the scenario was not feasible for actual STAR and D100, whereas the radius of $150 \mathrm{n}$ mile and cases with higher distances allowed synchronizing all arriving traffic successfully.

The date, 26 April 2016, was selected as the representative day with high arrival traffic intensity. On that day, there were 318 continuous arrivals through TEBLA approximately from 0600 to $1930 \mathrm{hrs}$. For the analyzed cases, 35, 29, 7, 5, and 4 subproblems were created, respectively. The time separations at TEBLA are presented in Fig. 10, and the arrival times at TEBLA for consecutive aircraft are presented in Fig. 11. Only for the D250 that all of the subproblems were solved. For the remaining cases, there existed one or more subproblems that were infeasible. It is also worth noticing that, for larger distances, the amount of infeasible subproblems was smaller than for shorter distances. For longer distances, the larger infeasible sequences (that are visible, e.g., for D150 and D200 in Fig. 11) were caused by the fact that subproblems were bigger and their amount was smaller than for shorter distances, because values of time windows increase with the distance. Therefore, even if for the D150 and D200 only a small dense sequence of aircraft appearing in the vicinity of $1700 \mathrm{hrs}$ was unable to schedule, it resulted in whole subproblem infeasibility.

For the scenario presented in Fig. 10 (high intensity), it was observed that even more flights were scheduled with a minimum separation value (approximately 67\%). From Figs. $\underline{6}, \underline{8}$, and $\underline{10}$, it can be observed that the number of flights with minimum separation increases with traffic density. It arises from the necessity of scheduling a greater amount of aircraft at the same time. Like for low and medium intensities, it was observed that less flights were performed with greater separation. Moreover, this decrease rate was highest in this scenario. Analogous to the number of flights with minimal separation, this results from the necessity of scheduling a higher number of aircraft at the same time.

By analyzing Figs. 10 and 11 , it can be observed that only the radius of $250 \mathrm{n}$ mile allowed for successful traffic synchronization. Nevertheless, the infeasibility of other cases is caused usually by small sequences of aircraft, while most part of traffic is synchronizable, and so still even a smaller radius may be applicable in a favorable initial aircraft distribution. On the other hand, an unfavorable distribution may cause infeasibility of all the cases. In this scenario, it can be noticed that the number of infeasible sequences decreases with distance. For STAR, D100, D150, and D200, there were, respectively, three, two, one, and one infeasible subproblems. By combining this notice with the fact that, in a moderate-traffic-intensity scenario there was only one short infeasible sequence for STAR and D100, it can be concluded that scenario infeasibility is caused most often by a small group of aircraft that were tightly distributed in time.

In the study, 16 days were analyzed. The data set was divided into days with low (5), moderate (10), and high (1) air traffic intensities, basing on arrival density defined as the number of arrivals divided by the time range. Table $\underline{3}$ presents the percentage of days for which all the flights were scheduled successfully for various distances. The STAR case was not presented in the table because its distance to the runway is not a constant value. From the table, it can be seen that,

Table 3 Summary of test scenario feasibility

\begin{tabular}{lcccc}
\hline \hline Intensity & $\mathrm{D} 100, \%$ & $\mathrm{D} 150, \%$ & $\mathrm{D} 200, \%$ & $\mathrm{D} 250, \%$ \\
\hline Low & 80 & 100 & 100 & 100 \\
Medium & 0 & 90 & 90 & 100 \\
High & 0 & 0 & 0 & 100 \\
\hline \hline
\end{tabular}


for low intensity, all cases were successfully synchronized when at least a distance of $150 \mathrm{n}$ mile was selected. For moderate intensity, $90 \%$ of days were scheduled successfully for this distance. For high intensity, only the D250 case allowed to schedule all the aircraft. However, it has to be underlined that just one day was assigned to this category, whereas $93.75 \%$ of simulated days had low or moderate intensity.

The results showed that extending TMAs can significantly improve the ability to sequence and merge arrival traffic with RTA, satisfying safety requirements and enabling CDOs. Even without TMA extension for days with low or moderate traffic, it may be possible to perform CDOs with assigned RTA. It was observed that assigning RTA at $150 \mathrm{n}$ mile could be sufficient for days with low arriving traffic. Extending TMA to $250 \mathrm{n}$ mile should be enough for scenarios with high-traffic and complex scenarios. The distance $250 \mathrm{n}$ mile is also considered as the radius value of future TMAs [36].

Summarizing the results from all scenarios, it can be concluded that the scenario feasibility rises with the rise of TMA radius and, for infeasible scenarios, a number of infeasible sequences decrease with the rise of TMA radius. Moreover, it is worth noticing that the number of subproblems decreases with the rise of TMA radius, which causes infeasible sequences to be significantly bigger than for cases with numerous subproblems.

\section{Conclusions}

Assigning required times of arrival (RTA) at a metering fix to each inbound aircraft has been identified as a potential solution to enable Continuous descent operations (CDOs) without compromising capacity. However, the feasibility of this time-based sequencing and merging technique deserves further research for high-traffic-density scenarios.

This paper assessed the feasibility and performance of replacing the current sequencing and merging techniques (mainly based on radar vectoring) by a control based on RTA. To accomplish that, the arrival traffic at Barcelona-El Prat Airport (Spain) has been gathered from historical data, and several days have been reproduced by assuming all aircraft following their standard terminal arrival routes (STARs), performing engine-idle CDOs, and being sequenced and merged at the intermediate fix by means of RTA.

A point-mass model of aircraft dynamics together with an nonlinear programming solver allowed for quick calculations of optimal trajectories, whereas an mixed-integer linear programming solver allowed for the successful solution of aircraft landing problem.

It was observed that, for low-traffic scenarios, an ideal arrival traffic with only CDOs could be handled without radar vectoring by assigning RTA at a metering fix to each inbound aircraft when arriving at the entry points of their corresponding STARs. As the traffic complexity increases, these RTA should be assigned well in advance to achieve a feasible scheduling. It can be seen from the results that notifying the RTA at $150 \mathrm{n}$ mile from the runway threshold would be sufficient for days with low traffic. Extending the RTA notification distance to $250 \mathrm{n}$ mile should be enough for scenarios with high traffic.

In the study, it was noticed that the majority of flights are performed with minimum possible time separation, regardless the distance. The number of those flights increased when the traffic intensity was higher.

Moreover, it has to be mentioned that, generally, the feasibility of the scenarios depends mainly on small sequences of aircraft, which were not able to be synchronized, and it was observed that the number of these sequences always decreases with rising distance to the airport where RTA are assigned to aircraft.

It is worth mentioning that the presented method is well suited for a closely spaced parallel runway pair where one runway is designated for takeoffs and the second one for landings. This layout allows achieving a sequence of consecutively approaching aircraft all day long that is favorable from a traffic-synchronization point of view. Also, crossing runways can be considered for direct use of this method, as long as the intersection point is located in the end of at least one of the runways, so it allows for independent landing operations. Contemporary biggest airports with high-traffic demand often have several runways and various layouts. Simultaneous arrival operations to multiple runways are performed on those airports. Therefore, a more complex approach is required in this case. This approach should consist of a detailed traffic analysis on the given airport and selection of methodology for handling arrivals to multiple runways, which can consist of extending an airport landing problem (ALP) by adding equations considering multiple runways as additional objectives or by assigning a target runway in advance. With the latter approach, the ALP presented in this study can be applied separately to every runway. The method can also be used for single runways and crossing runways with an intersection point close to the middle of both runways, for cases when a sequence of arriving aircraft exists. In this case, an additional constraint is required that limits the time window for the whole arriving aircraft sequence.

In the experimental scenario, only one merging point was considered to assess the feasibility of the method. However, this method can be extended to multiple merging points on a descent trajectory by solving the ALP repeatedly for the RTA in given merging points with an assumption that, for the subsequent merging point, the former one is the waypoint where RTA are assigned. Although the time windows in this case are expected to be smaller, as relative distances between RTA notification points and merging points are smaller compared to the approach with one merging point, the presented method still should be applicable for scenarios with low and moderate traffic demands.

The sequencing and merging approach based on the assignment of RTA analyzed in this paper would require decision support tools on ground for the controller (including advanced arrival managers) in addition to an air-ground protocol over a data link for the negotiation of RTA. Flight management systems with data-link technology and 4-D planning and guidance capabilities would be required as well.

Future work will focus on the use of multiple aircraft data to accurately reflect the dynamics of various aircraft. Also, large-scale simulation, which takes into account border cases with very-hightraffic intensity for different airport scenarios, should be performed, and the focus should be put on optimization of problems solving time. In further works also, the use of other algorithms to calculate trajectories and to synchronize air traffic may be considered to take into account the uncertainties or necessity to adapt to varying initial conditions. Genetic algorithms or particle swarm optimization, together with parallel computing, could be a potential alternative to the presented approach.

\section{Acknowledgment}

The authors would like to thank Airbus Industries for the use of Performance Engineering Program suite, which allowed them to undertake realistic aircraft performance simulations.

\section{References}

[1] Erkelens, L., "Research into New Noise Abatement Procedures for the 21st Century," AIAA Guidance, Navigation, and Control Conference and Exhibit, Guidance, Navigation, and Control and Co-located Conferences, AIAA Paper 2000-4474, Aug. 2000.

[2] Warren, A., and Tong, K., "Development of Continuous Descent Approach Concepts for Noise Abatement," Proceedings of the IEEE/ AIAA 21st Digital Avionics Systems Conference (DASC), IEEE Publ., Piscataway, NJ, 2002, pp. 1.E.3.1-1.E.3.4.

[3] Clarke, J. P. B., Ho, N. T., Ren, L., Brown, J. A., Elmer, K. R., Zou, K. F., Hunting, C., McGregor, D. L., Shivashankara, B. N., Tong, K., et al., "Continuous Descent Approach: Design and Flight Test for Louisville International Airport," Journal of Aircraft, Vol. 41, No. 5, 2004, pp. 1054-1066.

doi: $10.2514 / 1.5572$

[4] Robinson, J., and Kamgarpour, M., "Benefits of Continuous Descent Operations in High-Density Terminal Airspace Under Scheduling Constraints," 10th AIAA Aviation Technology, Integration, and Operations (ATIO), AIAA Paper 2010-9115, Sept. 2010.

[5] Klooster, J. K., Amo, A. D., and Manzi, P., "Controlled Time-of-Arrival Flight Trials," 8th USA/Europe Air Traffic Management Research and Development Seminar, Curran Associates, Red Hook, NY, 2009, pp. 219-229. 
[6] de Jong, P. M. A., Bussink, F. J. L., Verhoeven, R., de Gelder, N., Paassen, M. M. V., and Mulder, M., "Time and Energy Management During Descent and Approach: A Human-in-the-Loop Study," Journal of Aircraft, Vol. 54, No. 1, 2017, pp. 177-189. doi:10.2514/1.C033741

[7] Prats, X., Bendris, B., Dalmau, R., Montolio, J., Day, B., Lenz, H., and Kohrs, R., "4D Continuous Descent Operations Supported by an Electronic Flight Bag a Human-in-the-Loop Study," 35th IEEE/AIAA Digital Avionics Systems Conference (DASC), IEEE Publ., Piscataway, NJ, Sept. 2016.

[8] De Smedt, D., Bronsvoort, J., and McDonald, G., "Controlled Time of Arrival Feasibility Analysis," 10th USA/Europe Air Traffic Management Research and Development Seminar, Curran Associates, Reed Hook, NY, 2013, pp. 219-300.

[9] Houston, V. E., and Barmore, B., "An Exploratory Study of Runway Arrival Procedures: Time-Based Arrival and Self-Spacing," 9th AIAA Aviation Technology, Integration, and Operations Conference (ATIO), AIAA Paper 2009-7005, Sept. 2009.

[10] Park, S. G., and Clarke, J., "Optimal Control Based Vertical Trajectory Determination for Continuous Descent Arrival Procedures," Journal of Aircraft, Vol. 52, No. 5, 2015, pp. 1469-1480. doi:10.2514/1.C032967

[11] Takeichi, N., "Nominal Flight Time Optimization for Arrival Time Scheduling Through Estimation/Resolution of Delay Accumulation," Transportation Research Part C: Emerging Technologies, Vol. 77, April 2017, pp. 433-443. doi:10.1016/j.trc.2017.01.025

[12] Dalmau, R., and Prats, X., "Assessment of the Feasible CTA Windows for Efficient Spacing with Energy-Neutral CDO," Proceedings of the 7th International Conference on Research in Air Transportation (ICRAT), Federal Aviation Administration/Eurocontrol, 2016, Paper 9.

[13] Faye, A., "Solving the Aircraft Landing Problem with Time Discretization Approach," European Journal of Operational Research, Vol. 242, No. 3, 2015, pp. 1028-1038. doi:10.1016/j.ejor.2014.10.064

[14] Preisighe Viana, M. V., "Time-Domain System Identification of Rigid Body Multipoint Loads Model," AIAA Atmospheric Flight Mechanics Conference, AIAA Paper 2016-3706, June 2016.

[15] Fischenberg, D., Mönnich, W., Krag, B., and Jategaonkar, R. V., "Aspects of C-160 Simulator Model Determination and Validation on and Close to the Ground," AIAA Flight Simulation Technologies Conference, AIAA Paper 1994-3404, Aug. 1994, pp. 22-31.

[16] Seren, C., Bommier, F., Bucharles, A., Verdier, L., and Alazard, D., "Flight Test Protocol Optimization Using Genetic Algorithms," 14th IFAC Symposium on System Identification-SYS ID'06, Curran Associates, Red Hook, NY, 2006, pp. 642-647.

[17] Lichota, P., and Ohme, P., "Design and Analysis of New Multi Axis Input Manoeuvres for Aircraft Sys-ID," DLR, German Aerospace Center, IB 111-2014/46, Braunschweig, Germany, Nov. 2014.

[18] Anon., "A320 Aircraft Characteristics Airport and Maintenance Planning," Blagnac Cedex, France, May 2016, https://www.airbus.com/ content/dam/corporate-topics/publications/backgrounders/techdata/
aircraft_characteristics/Airbus-Commercial-Aircraft-AC-A320-Feb18. pdf [retrived 24 July 2018].

[19] Stengel, F., Flight Dynamics, Princeton Univ. Press, Princeton, NJ, 2004, pp. 147-158.

[20] Vilardaga, S., and Prats, X., "Operating Cost Sensitivity to Required Time of Arrival Commands to Ensure Separation in Optimal Aircraft 4D Trajectories," Transportation Research Part C: Emerging Technologies, Vol. 61, Dec. 2015, pp. $75-86$ doi:10.1016/j.trc.2015.10.014

[21] Dalmau, R., and Prats, X., "Fuel and Time Savings by Flying Continuous Cruise Climbs Estimating the Benefit Pools for Maximum Range Operations," Transportation Research Part D: Transport and Environment, Vol. 35, March 2015, pp. 62-71. doi:10.1016/j.trd.2014.11.019

[22] Betts, J. T., Practical Methods for Optimal Control and Estimation Using Nonlinear Programming, 2nd ed., Soc. for Industrial and Applied Mathematics, Philadelphia, PA, 2010, pp. 153-165.

[23] Bryson, A. E., and Ho, Y.-C., Applied Optimal Control: Optimization, Estimation, and Control, Taylor and Francis, New York, 1975, pp. 1-41.

[24] Gueret, C., Prins, C., and Sevaux, M., Applications of Optimization with Xpress-MP, Dash Optimization, Paris, 2007, pp. 157-173.

[25] McCarl, B. A., and Spreen, T. H., Applied Mathematical Programming Using Algebraic Systems, Texas A\&M Univ., College Station, TX, 2011, pp. 1-10.

[26] Anon., "DDR2 Reference Manual for Generic Users 2.1.2," Brussels, Belgium, June 2015, pp. 13-22.

[27] Anon., "Barcelona/El Prat plano de aerodrómo-oaci," ENAIRE, Madrid, Spain, Jan. 2016.

[28] Anon., "Barcelona/El Prat AD Standard Instrument Arrivals (STAR) Runway 25R/25L (West Configuration)," ENAIRE, Madrid, Spain, Oct. 2013.

[29] Anon., Eurocontrol Specification for the Electronic Aeronautical Information Publication (eAIP), Brussels, Belgium, Oct. 2015, pp. 99-100.

[30] Anon., NEST User Guide 1.5, Brussels, Belgium, 2016, pp. 1121-1124.

[31] Anon., "Flight Crew Operation Manual (FCOM)," A320, Ver. 1.3.1, Airbus, Blagnac Cedex, France, 1993.

[32] Drud, A., "CONOPT," ARKI Consulting and Development A/S, Bagsvaerd, Denmark, March 2010, https://www.pik-potsdam.de/ research/sustainable-solutions/models/remind/conopt.pdf [retrieved 24 July 2018].

[33] Stridsman, L., "SESAR Concept of Operations Step 1," SESAR Joint Undertaking, Brussels, Belgium, 2012.

[34] Holmström, K., Göran, A. O., and Edvall, M. M., User's Guide for TOMLAB/CPLEX v12.1, Tomlab Optimization, Västerås, Sweden, Aug. 2009, pp. 75-104.

[35] Park, S. G., and Clarke, J. P. B., "Optimal Control Based Vertical Trajectory Determination for Continuous Descent Arrival Procedures," Journal of Aircraft, Vol. 52, No. 5, 2015, pp. 1469-1480. doi:10.2514/1.C032967

[36] Ky, P., "SESAR Release 1 Results," SESAR Joint Undertaking, Brussels, Belgium, 2012. 